www.nature.com/jhg

\title{
EDITORIAL
}

\section{In the new year}

\author{
Journal of Human Genetics (2011) 56, 1-3; doi:10.1038/jhg.2010.158
}

$\mathrm{T}_{\mathrm{t}}^{\mathrm{h}}$ he year 2010 will be remembered as a year of steady progress for the Journal of Human Genetics (JHG). We could see positive results from the changes and developments that JHG underwent in 2009 with our new partner Nature Publishing Group. Not only did we see increases in the number of submissions $(+40 \%$ compared with last year) and increases in usage, we also witnessed the quality of submitted papers getting better than ever before. We are very pleased to see these changes and improvements.

In response to the considerable increase in submissions, we now need to expand the journal's editorial board. In particular, we need to expand the board of associate editors to handle the larger number of papers that need peer review, and to avoid taking too long time to process and judge each manuscript. Our main aim is to maintain and even improve the current 'speed' and 'quality' in judging and publishing papers, while attracting more papers from all parts of the world.

I am also proud that we were able to start publishing commentary articles in 2010. Commentaries are solicited to introduce the research or topics that are dealt with in other articles in the journal from a third person's viewpoint. I believe that these commentaries add another dimension to the journal, providing readers with a good introduction to the topics discussed and enhancing the impact of the articles that are being commented on. Although we are yet to establish a workflow to enable the regular publication of commentary papers,
I am confident that the number of commentary papers will also increase, and that this will prove to be a very important section in JHG in near future. I hope readers have fun reading JHG's commentary articles.

These improvements that we have seen throughout the last year, together with the changes we are still working on, should form solid ground for the JHG to stand on. From here, on this ground, we would like to build the journal's new, successful profile. With the Associate Editors and Editorial Board members, and together with the authors and readers, I am committed to continue to work hard and serve readers better by providing an important and authoritative source of information and an exciting forum for scientific communication.

Best wishes for the New Year.

Katsushi Tokunaga Editor-in-Chief

Journal of Human Genetics

\section{ACKNOWLEDGEMENTS}

The Editor-in-Chief, on behalf of the JHG Editorial Board and the Japan Society of Human Genetics, gratefully acknowledges the generous support from peer reviewers in carefully assessing manuscripts under consideration. The following individuals provided reviews of papers submitted to the JHG in 2010:

\author{
Abe, Jun \\ Aburatani, Hiroyuki \\ Akao, Yukihiro \\ Aksentijevich, Ivona \\ Anney, Richard JL \\ Aoki, Yoko \\ Arai, Makoto \\ Arima, Takahiro \\ Arinami, Tadao \\ Asano, Koichiro \\ Bae, Joon Seol \\ Bhatnagar, Vibha \\ Boffetta, Paolo \\ Bonne, Gisele \\ Boor, Ilja \\ Burzyńska, Beata \\ Caglayan, Ahmet Okay \\ Capasso, Mario
}

Carlson, Chris
Cha, Seongwon
Chaabani, Hassen
Chaiyaratana, Nachol
Chen, Xiangning
Cheong, Hyun Sub
Cherny, Stacey S
Chikhi, Lounès
Chung, SukJae
Clarke, Angus
Cox, Diane W
Dai, Pu
del Castillo, Ignacio
Desnick, Robert J
Diatchenko, Luda
Doi, Nagafumi
Economopoulos, Konstantinos P
Egly, Jean

\author{
Emre, Serap \\ Esteban, Esther \\ Estevez, Raul \\ Ezura, Yoichi \\ Ferguson-Smith, Anne \\ Fogli, Anne \\ Forman, Julia \\ Fujisawa, Tomomi \\ Fujita, Kenichi \\ Fukami, Maki \\ Fukumaki, Yasuyuki \\ Fukushima, Yoshimitsu \\ Furuta, T \\ Gambardella, Antonio \\ Gao, Peisong \\ Georgiou, Ioannis \\ Goizet, C \\ Goto, Jun
}

\author{
Goto, Yu-ichi \\ Grossfeld, Paul \\ Guerrini, Renzo \\ Guichaoua, MR \\ Hadano, Shinji \\ Hagiwara, Koichi \\ Hamada, Hiromichi \\ Hamajima, Nobuyuki \\ Hashimoto-Tamaoki, Tomoko \\ Hayashi, Shin \\ Hayashi, Yukiko \\ Hayden, Catherine M \\ Heron, Sarah \\ Hiratsuka, Masahiko \\ Honda, Shozo \\ Hong, Xiumei \\ Horikawa, Yukio \\ Hotta, Kikuko
}


Iida, Shinsuke Iijima, Kazumoto Iijima, Yoshimi Ikegawa, Shiro Ikeuchi, Takeshi Imoto, Issei Inoue, Hideyuki Inoue, Ituro Inoue, Ken Ionita-Laza, Iuliana Ishikawa, Kinya Ishikawa, Shumpei Isomura, Minoru Iwamoto, Sadahiko Iwasaki, Naoko Jama, Abdalla Jezewski, Peter Jobling, Mark Johnson, Todd Johnson, Matthew P Kagami, Masayo Kaito, Hiroshi Kamatani, Kengo Kamatani, Yoichiro Kamide, Kei Kamimura, Naomi Kampuansai, Jatupol Kanai, Yoshiakira Kaname, Tadashi Karami, Sara Katagiri, Toyomasa Kato, Mitsuhiro Kato, Norihiro Katoh, Toru Katsumata, Noriyuki Katsuya, Tomohiro Kawame, Hiroshi Kawano, Yuji Kazazian, Haig Kim, Sangsoo Kim, Un-kyung Kim, Jeong Hyun Kim, Young Joo Kimura, Akinori Kimura, Ryosuke Klungel, Olaf Kobayashi, Kazuhiro Kobayashi, Takehiro Kochi, Yuta Kohda, Takashi Kohno, Takashi Koizumi, Akio Kondo, Eri Kraemer, Kenneth $\mathrm{H}$ Kubo, Michiaki Kubota, Takeo

Kudoh, Jun Kurahashi, Hiroki Kure, Shigeo Kuroki, Yoko Kurosawa, Kenji
Lackner, Mark

Landsberger, Nicoletta

Lee, Heon-Jeong

Lee, Jong-Keuk

Lee, $\mathrm{P}$

Lee, Richard

Lerner-Ellis, Jordan

Limpiti, Tulaya

Lin, Hui-Yi

Liu, Mugen

Liu, Jian-Jun

Ma, Sai

Mabuchi, Akihiko

Maeda, Shiro

Mahasirimongkol, Surakameth

Makishima, Tomoko

Makita, Yoshio

Malerba, Giovanni

Mano, Shuhei

Masuda, Ryuichi

Masui, Toru

Matsubara, Yoichi

Matsumoto, Naomichi

Matsuo, Hirotaka

Matsuo, Keitaro

Matsuura, Masaaki

Matsuura, Tohru

Maximo, Valdemar

McLeod, Howard

Mehra, Narinder

Meroni, Germana

Metspalu, Mait

Michelsen de Andrade, Fabiana

Michigami, Toshimi

Migeon, Barbara

Miki, Tsuneharu

Millan, Jose

Minami, Narihiro

Miura, Kiyonori

Miyake, Noriko

Miyamoto, Toshinobu

Miyata, Satoshi

Miyata, Toshiyuki

Mizuta, Ikuko

Morisaki, Takayuki

Morrisey, Edward

Muramatsu, Masaaki

Mushiroda, Taisei

Mutirangura, Apiwat

Muzzio, Marina

Nakagawa, Eiji

Nakagawa, Masanori

Nakajima, Toshiaki

Nakamura, Akinori

Nakamura, Takahiro

Nakayama, Tomohiro

Nanba, Eiji

Narita, Akira

Nathanson, Katherine

Nishimura, Gen

Nishino, Ichizo
Noguchi, Emiko

Numabe, Hironao

O’Dell, Sandra

Ogata, Tsutomu

Ogimoto, Akiyoshi

Oh, Bermseok

Ohashi, Jun

Ohashi, Toya

Ohashi, Wataru

Ohishi, Mitsuru

Okada, Yukinori

Okamoto, Nobuhiko

Okano, Yoshiyuki

Okuyama, Torayuki

Omori, Kenji

Onodera, Osamu

Onouchi, Yoshihiro

Onuma, Hiroshi

Osada, Naoki

Osawa, Haruhiko

Ota, Masao

Ozaki, Kouichi

Ozaki, Norio

Ozawa, Nobuaki

Pan, Hong

Park, Choon-Sik

Park, Ji Wan

Paschou, Peristera

Pasha, MA

Piriyapongsa, Jittima

Praphanphoj, Verayuth

Price, Thomas

Ramachandra, Nallur B

Randhawa, Gursharn Singh

Ravn, Kirstine

Reitsma, P

Roux, Anne-Francoise

Rubes, J

Saitoh, Shinji

Saitou, N

Saitou, Naruya

Saitsu, Hirotomo

Sakamoto, Osamu

Sakurai, Akihiro

Sanjay, Shete

Sasaki, Hidenao

Satake, Wataru

Sato, Fumiaki

Sato, Shinichi

Sato, Yoshiaki

Sawamura, Daisuke

Schelleman, Hedi

Schosser, Alexandra

Schultz, Julie

Seki, Naohiko

Serretti, Alessandro

Shaw, Philip

Shibata, Kyoko

Shimizu, Norikazu

Shin, Dong-Jik

Shotelersuk, Vorasuk
Smigiel, Robert

Snyder, Eric M

Sobrinho-Simos, M

Sobue, Gen

Soejima, Hidenobu

Soejima, Mikiko

Song, Kyuyoung

Stankovich, Jim

Stein, Catherine

Stoneking, Mark

Su, Bing

Sugano, Kokichi

Suktitipat, Bhoom

Suzuki, Fumihiko

Suzuki, Hiroshi

Suzuki, Hiroyuki

Suzuki, Yoichi

Suzuki, Akari

Tabara, Yasuharu

Tajima, Atsushi

Tajima, Toshihiro

Takada, Fumio

Takahashi, Atsushi

Takahashi, Yuji

Takano, Hiroki

Takashima, Hiroshi

Takeuchi, Fumihiko

Tamaki, Shigehiro

Tamari, Mayumi

Tamiya, Gen

Tamura, Kazuo

Tanaka, Akemi

Tanaka, Kenichi

Tanaka, Masashi

Tanaka, Toshihiro

Tanaka, Yasuhito

Tang, Tiong Yang

Taniguchi, Atsuo

Teo, YY

Teraoka, Hirobumi

Thangaraj, Kumarasamy

Tokunaga, Katsushi

Tomatsu, Shunji

Tomiyama, Hiroyuki

Toniolo, Daniela

Tsezou, Aspasia

Tsuchiya, Naoyuki

Tsujikawa, Motokazu

Usami, Shinichi

Vicente, Astrid

Wada, Takahito

Wallace, RH

Watanabe, Mikio

White, Robert

Wilder, Jason A

Wirtz, Mary

Woo, Sung-Il

$\mathrm{Xu}$, Shuhua

Yamada, Ryo

Yamada, Yoshiji

Yamagishi, Hiroyuki 
Yamaguchi-Kabata, Yumi

Yamamoto, Ken

Yamamoto, Midori

Yamamoto, Toshiyuki

Yamauchi, Toshimasa
Yang, Zhenling

Yao, Yong-Gang

Yasunami, Michio

Yokota, Mitsuhiro

Yoshida, Kiyotsugu
Yoshida, Kunihiro

Yoshiura, Koh-ichiro

Yuasa, Yasuhito

Zaitlen, Noah

Zhang, Kui
Zheng, Minghao

Zhou, Hui

Zuchner, Stephan 